\title{
A new generation of arachidonic acid analogues targeting cytosolic phospholipase A2
}

\author{
Chian-Ming Low ${ }^{1,2}$, Cheng Yang $\mathrm{Ng}^{3}$, Srinivasaraghavan Kannan ${ }^{4}$, Yong Jun Chen ${ }^{5}$, Francis Chee \\ Kuan $\mathrm{Tan}^{2}$, Wee Yong Ong ${ }^{3}$, Mei Lin Go ${ }^{5}$, Chandra S. Verma ${ }^{4}$, Yulin Lam ${ }^{3}$ \\ ${ }^{I}$ Department of Pharmacology, Yong Loo Lin School of Medicine, National University of Singapore, Singapore, \\ ${ }^{2}$ Department of Anaesthesia, Yong Loo Lin School of Medicine, National University of Singapore, Singapore, \\ ${ }^{3}$ Department of Chemistry, Faculty of Science, National University of Singapore, Singapore, ${ }^{4}$ Bioinformatics Institute, \\ Agency for Science, Technology and Research (A*STAR), Singapore, Singapore, ${ }^{5}$ Department of Pharmacy, Faculty of \\ Science, National University of Singapore, Singapore
}

Cytosolic phospholipase A2 (cPLA2) is an enzyme that releases arachidonic acid for the synthesis of eicosanoids and lysophospholipids which play critical roles in the initiation and modulation of oxidative stress and neuroinflammation. In the central nervous system, cPLA2 activation is implicated in the pathogenesis of various neurodegenerative diseases that involves neuroinflammation, thus making it an important pharmacological target. We present (a) a new class of arachidonic acid analogues and their ability to inhibit cPLA2 and (b) arachidonic acid derivatives equipped with either one or two fluorescent groups attached to the tip of the alkyl chains that function as inhibitor and substrate probes of cPLA2. Several compounds inhibit cPLA2 more strongly than arachidonyl trifluoromethyl ketone (AACOCF3), an inhibitor that is commonly used in the study of cPLA2-related neurodegenerative diseases. One of the inhibitors was found to be cPLA2-selective, non-cytotoxic, cell and brain penetrant and capable of reducing reactive oxygen species (ROS) and nitric oxide (NO) production in stimulated microglial cells. Computational studies shed insights on how the compound interacts with cPLA2. The fluorogenic inhibitor probe exhibited dual functions of inhibition and imaging while the fluorogenic substrate probe could be used for activity assay. 